\title{
Snow effect on the neutron monitor network for 2018-2019
}

\author{
Pavel G. Kobelev ${ }^{\circledR 1}$, Liudmila Trefilova', Lev I. Dorman ${ }^{1,2}$, Lev A. Pustilnik ${ }^{2}$, Anatoly V. Belov ${ }^{\circledR 1}$, \\ Eugenia A. Eroshenko ${ }^{\oplus 1}$, Victor G. Yanke \\ Correspondence \\ 'Pushkov Institute of Terrestrial Magnetism, Ionosphere and Radio Wave Propagation (IZMIRAN), Moscow, Russia, \\ kosmos061986@yandex.ru, trefilova@izmiran.ru, lid010529@gmail.com, abelov@izmiran.ru, \\ erosh@izmiran.ru, yanke@izmiran.ru \\ ${ }^{2}$ Israel Cosmic Ray and Space Weather Center, Tel Aviv University, Israel, \\ levpust2149@gmail.com
}

\section{OPEN ACCESS}

This work is published under the Creative Commons Attribution 4.0 International licence (CC BY 4.0). Please note that individual, appropriately marked parts of the work may be excluded from the licence mentioned or may be subject to other copyright conditioj. If such third condy m. if such thirdparty material is not under the Creative Commons license, any copying, editing or public reproduction is only permitted with the prior consent of the respective copy right owner or on the basis of relevan legal authorization regulations.

\section{Keywords}

snow effect; neutron monitor; variations; barometric effect

\begin{abstract}
In this article, the influence of the surrounding snow cover on the neutron monitors count rate of the world network of neutron monitors was estimated using the method of reference stations. The applied technique also makes it possible to estimate the snow cover thickness at the observation point, which was done for more than two dozen stations. A comparison of the data correction results for snow is carried out for the case of automatic correction, based on the developed algorithm, and for manual one, with an error estimate.
\end{abstract}

\section{Introduction}

For some stations, especially mountain ones, snow is a big problem, because due to high humidity it effectively accumulates above and around the detector. For most of these stations, it is not possible to mechanically remove the snow. Therefore, the monitoring data for the neutron component, which are significantly distorted by a variable snow layer, are not suitable for studying many types of variations and require appropriate correction for further use. The registration accuracy of the 18 NM64 neutron monitor is about $0.15 \%$ for the hourly-averaged interval. And already one centimeter of thick snow $0.5 \mathrm{~cm}$ of water equivalent above the detector leads to a distortion of the observed variations by $0.5 \%$. Indeed, the effect is approximately determined by multiplying the barometric coefficient $(\sim 0.7 \% / \mathrm{hPa})$ on the absorber thickness.

The nature of the snow effect is twofold. The snow cover above the detector is an additional absorber, and this leads to a decrease of count rate. In addition, the neutron monitor registers a certain fraction of neutrons that are generated in the substance surrounding the detector, in particular in the ground. The snow cover shields this neutron source, which also leads to count rate decreasing. The effect of snow has been considered in many works, for example, (Korotkov et al. 2011, 2013), which also review earlier works.

$\dagger$ deceased 


\section{Data and method}

We have learned how to exclude the barometric effect, which has a similar nature, by using precision measurements of atmospheric pressure. Therefore, it is possible to make appropriate corrections by measuring the thickness of the snow cover. Indeed, if, in the absence of snow, the counting rate of the detector is $N_{i}^{\text {cor }}$, then the counting rate of the detector due to absorption with some effective range $L$ (assuming that $L$ does not depend on energy) in the snow depth $x_{i}$ is equal to $N_{i}=N_{i}^{\text {cor }} \cdot \exp \left(-x_{i} / L\right)$. Thus, the restored count rate

$$
N_{i}^{\text {cor }}=N_{i} / \varepsilon_{i} \text {, where } \varepsilon_{i}=\exp \left(-x_{i} / L\right)
$$

where $\varepsilon_{i}$ can formally be considered a change of the detector efficiency, i.e. a change in some properties of a detector or observation conditions. If we knew the thickness of the snow cover, then the data could be easily corrected for the effect of snow (Blomster \& Tanskanen 1969). But precise data on the thickness of the snow cannot be obtained due to the inaccessibility of the stations. At the same time, we are always talking about a certain effective thickness of the snow cover, which accumulates around the detector in the most bizarre way. On the sunny side, the snow melts faster, thereby further increasing the snow mass's unevenness near the detector. Therefore, we need to look for other approximate methods. One of them is based on comparing the variations recorded at the station under consideration with the variations without snow (considered as reference). This is not an ideal solution, since a detector surrounded by snow has slightly different coupling functions than a reference one, but this method is a fairly good approximation, as the practical application has shown.

Based on (1), the variations $v_{i}^{\text {cor }}$ of count rate at detector $i$ relative to the base count rate value $N_{B}$, corrected for the snow effect and expressed in terms of the measured variations $v_{i}$ can be written as

$$
v_{i}^{\mathrm{cor}}=\frac{N_{i}^{\mathrm{cor}}}{N_{B}}-1=\frac{N_{i} / \varepsilon_{i}}{N_{B}}-1=\frac{v_{i}+1}{\varepsilon_{i}}-1
$$

It can be seen from (2) that in order to determine the snow-corrected variations $v_{i}^{\text {cor }}$ from the measured variations $v_{i}$, it is necessary to evaluate the efficiency $\varepsilon_{i}$. For this purpose, we will use the data of the reference detector with count rate $S$, which records approximately the same variations $v^{S}$ as the detector exposed to the influence of snow $v^{\text {cor }}$, i.e. $v^{S} \simeq v^{\text {cor }}$ The selection criterion for a reference detector is discussed below. If this condition is applied to some averaged time interval, then we can write that

$$
\frac{\bar{S}}{S_{B}}-1=\frac{\bar{N} / \varepsilon}{N_{B}}-1 \text { or } \varepsilon=\frac{\bar{N} / N_{B}}{\bar{S} / S_{B}}=\frac{\bar{v}+1}{\bar{v}^{S}+1}
$$

When determining the average values of counting rate $\bar{N}, \bar{S}$ and accordingly variations $\bar{v}, \bar{v}^{S}$ $\left(S_{B}\right.$ is the value of reference detector's counting rate), the averaging interval is also important. We applied a filter of moving average (Smith 2002). The optimal filter length is $n=23$. If one-way filters are applied, then this technique can be applied in real time (Smith 2002).

In an ideal case, the detectors are identical and located at the same point, which guarantees the same variation. The selection of a closely located reference station is not always possible, since, as we will see, almost all mid- and high-latitude stations are affected by snow. A universal, although somewhat laborious, is the estimation of the expected variations based on the analysis of the world neutron monitor network data.

In other cases, it is necessary to take into account their differences, using the reception coefficients $C_{0}$ of these detectors (Kobelev et al. 2011, 2013). Variations for each detector in the 
zero harmonic approximation can be written as $\boldsymbol{v}^{S}=a_{10} C_{0}^{S}$ and $\boldsymbol{v}=a_{10} C_{0}$, so $\boldsymbol{v}^{S} / C_{0}^{S}=\boldsymbol{v} / C_{0}$. Considering that

$$
\left(\frac{\bar{S}}{S_{B}}-1\right) \frac{1}{C_{0}^{S}}=\left(\frac{\bar{N} / \varepsilon}{N_{B}}-1\right) \frac{1}{C_{0}},
$$

instead of (3) we get

$$
\varepsilon=\frac{\bar{v}+1}{C_{0} / C_{0}^{S} \cdot \bar{v}^{S}+1}
$$

The final corrections from the effect of snow variations in the detector should be carried out using expression (2), which involves the efficiency $\varepsilon$ obtained from equation (5). The receiving coefficients of the zero harmonic for some detectors (lines 1 and 2), which must be freed from the snow effect and for the reference stations involved (lines 3 and 4), are given in table 1.

\begin{tabular}{|l|l|l|l|l|l|l|l|}
\hline ESOI & Magadan & Moscow & Jungfraujoch & AlmaAta & LomnitskyStit & Nain & Peawanuck \\
\hline 0.4324 & 1.0044 & 0.9331 & 0.8924 & 0.6442 & 0.9113 & 1.1195 & 1.1194 \\
\hline Rome & Mexico & Thailand & Jungfraujoch1 & Athens & Potchefstrom & Tsumeb & Kiel \\
\hline 0.5440 & 0.4518 & 0.2815 & 0.8924 & 0.4360 & 0.5383 & 0.4406 & 0.9505 \\
\hline
\end{tabular}

Table 1: The receiving coefficients of the zero harmonic for some detector.

Several options for a reference station were considered. The Rome reference station option is chosen as the best (guaranteed there is no snow, long observation range, stable operation, good statistics of the $17 \mathrm{~nm} 64$ detector).

\section{Discussion of the results}

The effective thickness of the snow cover at the ESOI station reaches $15 \mathrm{~g} / \mathrm{cm}^{2}$ (middle panel of Figure 1 - right). Effective snow depth is formed from the snow on the surface of the Faraday cage
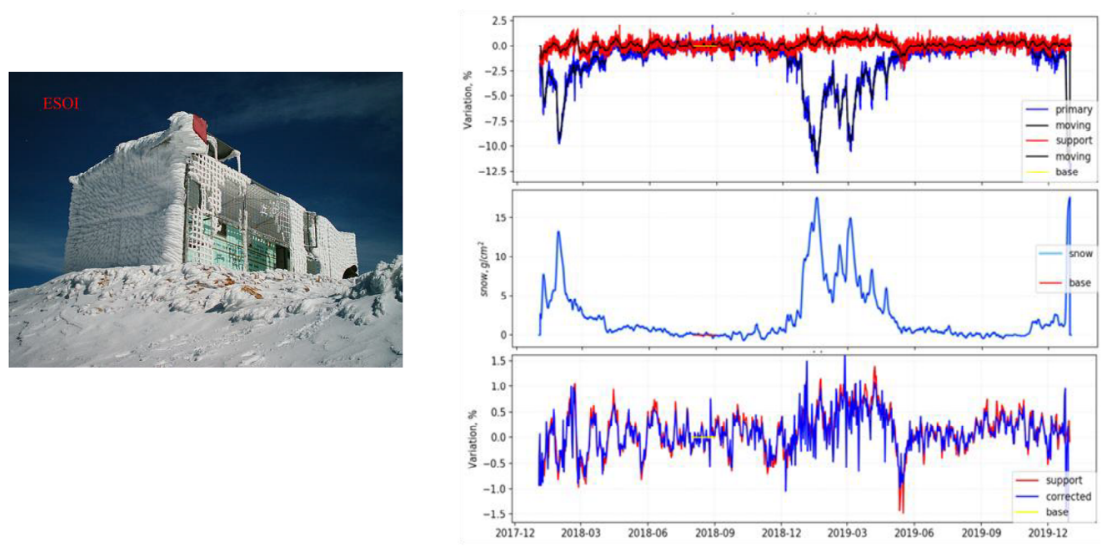

Figure 1: Station ESOI and snow effect: comparing of uncorrected and corrected variation (upper panel), snow thickness (middle panel), comparing the variation of ESOI (blue line) and Rome (red line) for 2018-2019 (bottom panel). 
and the snow surrounding the station. It can be assumed that the notches in the middle panel in figure 1 are associated with the periodic growth and melting of snow on the surface of the Faraday cage, and also that $1 / 3$ of the effect is due to the snow surrounding the station.

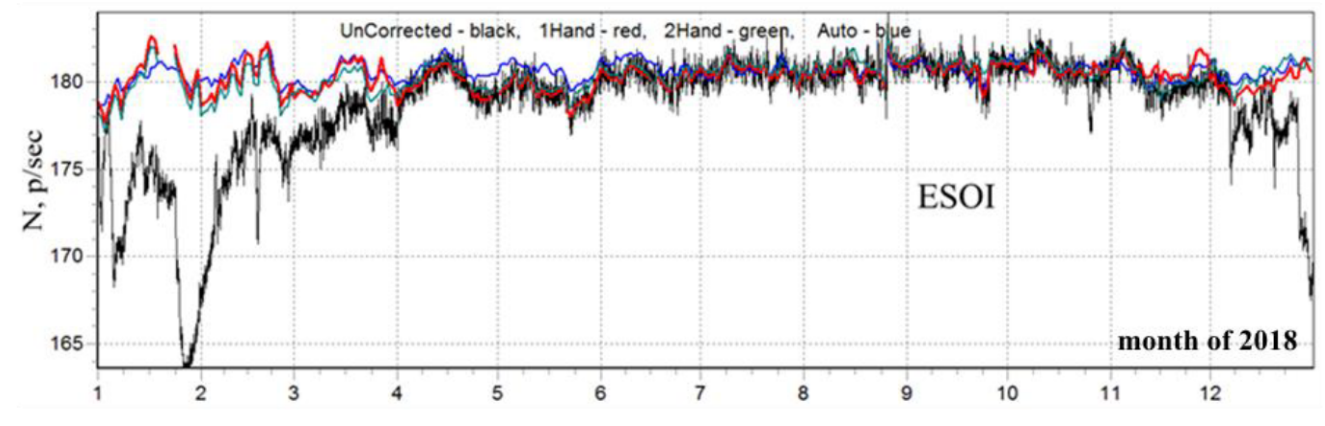

Figure 2: Comparing manually and automatically corrected data.

Figure 2 compares data adjusted by the method under discussion and data adjusted manually by two independent operators. The spread averaged over a year is no more than $0.1 \%$.

From mid-latitude stations (Moscow, Novosibirsk, Magadan, Irkutsk, Peawanuck, Nain), one can consider the Moscow station. Despite the fact that the detector at the station is located in a building with a hipped roof, the effective snow thickness reaches $2 \mathrm{~cm}$ w.e. (figure 3 ), and the contribution from each of the 4 sections of the neutron monitor is the same. This indicates that the collection of neutrons occurs from a sufficiently large area and the unevenness of its coverage is imperceptible. The meteorological data can be found on the resource (Ventusky 2020).

High-latitude cosmic ray stations can be divided into two groups. The stations of the first group are close to mid-latitude detectors in terms of the snow effect. The effective thickness of the accumulated snow for such stations is $2-3 \mathrm{~cm}$ w.e. and they are located in the polar latitudes, where the
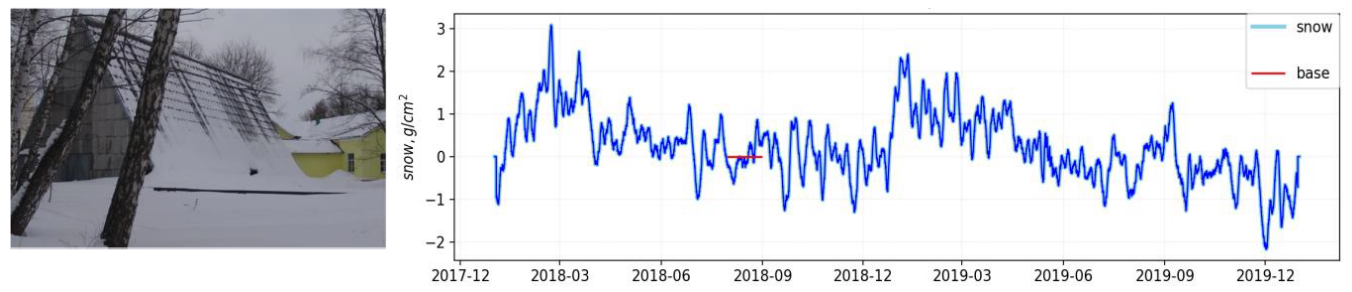

Figure 3: Station Moscow and effective snow thickness. Reference station is Rome

humidity of the air of the Gulf Stream is felt. Due to special conditions, this group also adjoins the Antarctic stations: Mirny (Fig.4), Terre Adelie, Mawson, YanBogo, Concordia, Sanae.

High-latitude stations of the second group are located in an area with sufficiently low humidity, where dry snow accumulates less on the roof and near the stations. These stations include Norilsk, Apatity, Tixie Bay, Cape Schmidt, Inuvik, where the snow effect is insignificant and close to the method error, i.e. $0.5 \mathrm{~cm}$ w.e. during the winter period. 

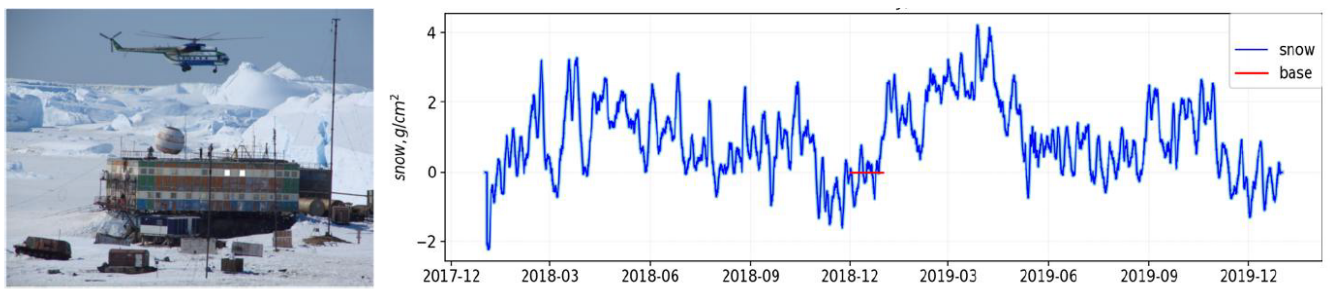

Figure 4: Station Mirny and effective snow thickness. Reference station is Rome.

The influence of snow cover near the detectors on their count rate was studied. Snow affects the data of all mountain, mid-latitude and most high-latitude detectors, which can be divided into groups.

For mountain stations, the effective thickness of the snow cover in winter reaches $10 \mathrm{~cm}$ of water equivalent (cm w.e.), which leads to significant errors of observed variations of cosmic rays, up to $10 \%$ (figure 5 ).

\section{Conclusions}

The influence of snow cover near the detectors on their count rate was studied. Snow affects the data of all mountain, mid-latitude and most high-latitude detectors, which can be divided into groups.

For mountain stations, the effective thickness of the snow cover in winter reaches $10 \mathrm{~cm}$ of water equivalent (cm w.e.), which leads to significant errors of observed variations of cosmic rays, up to $~ 10 \%$.

For mid-latitude and high-latitude stations, despite the use of hipped roofs, the effective thickness of the snow cover reaches about $2 \mathrm{~cm}$ w.e., which leads to errors of the observed variations in cosmic rays by up to $3 \%$.

However, some of the high-latitude stations are located in areas with sufficiently low humidity, and dry snow accumulates less on the roof and near the stations. These stations include Norilsk, Tixie Bay, Cape Schmidt, Inuvik, Fort Smith.

The method of reference stations used in this work makes it possible to recover actual data almost automatically, by excluding the effect of snow influence. The error, in this case, depends on the thickness change rate of the snow cover (the characteristic time is a day). If the changes are slow (several days), then the errors introduced during data recovery can be neglected. If the
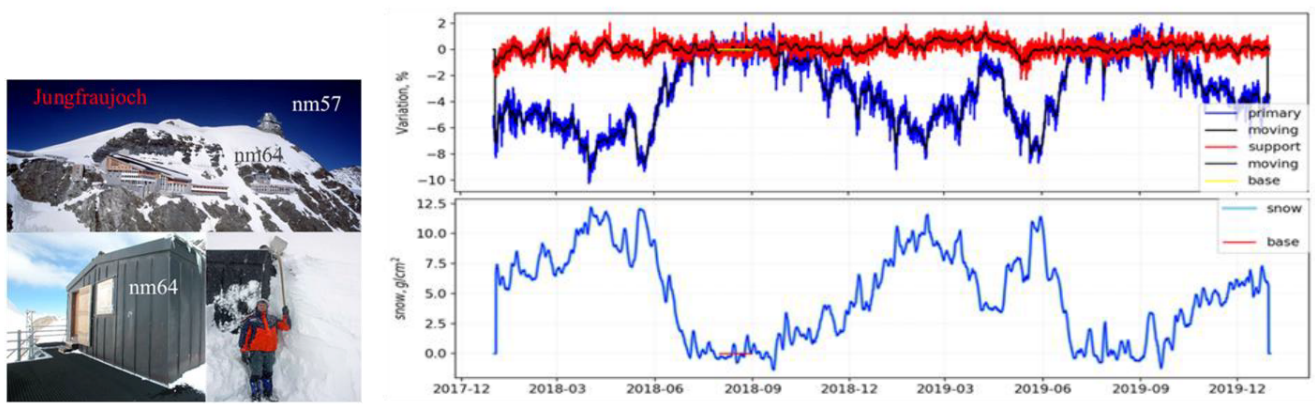

Figure 5: Station Jungfraujoch and effective snow thickness. Primary - station Jungfraujoch; reference (support) station - Rome; black lines - result of moving average filter. 
changes are fast (several hours), then the errors that arise should be investigated especially, since they can increase the errors of the original data several times. But rapid changes are rare - only in moments of heavy snowfall, intense snow melting, or mechanical snow removal. The technique described in this work makes it possible not only to exclude the effect of snow, but also to estimate the effective thickness of the snow cover with an accuracy of $0.5 \mathrm{~cm}$ w.e.

The reference station method is good for solving the problem of automatic excluding of the surrounding snow cover influence on the counting rate of neutron detectors. But it is almost impossible to find an ideal reference station (closely located, snowless, working without interruptions during the period under consideration). Therefore, in order to ensure the guaranteed absence of snow, it is necessary to use mid-latitude or even low-latitude stations and introduce corrections for isotropic variations using the method of reception coefficients.

\section{Acknowledgments}

This work was partially supported by the grant RFBR № 18-02-00451. Experimentally and methodologically support the project USU „Russian national network of ground stations of cosmic rays“. We are grateful to all the staff of the World Network of cosmic ray stations http://cr0.izmiran.ru/ ThankYou. We acknowledge the NMDB database (https://www.nmdb.eu/), founded under the European Union's FP7 programe (contract no. 213007) for providing the data.

\section{References}

Blomster, K., Tanskanen, P., 1969. "The influence of snow and water on the different multiplicities as observed in a neutron monitor NM-64 in Oulu", Proceedings of the $11^{\text {th }}$ ICRC, Budapest, Vol. 2. Acta Physica, Supplement to Volume 29, 627

Kobelev, P., Belov, A., Mavromichalaki, E., Gerontidou, M., Yanke, V., 2011, "Variations of Barometric Coefficients of the Neutron Component in the 22-23 Cycles of Solar Activity", Proc. 32 ${ }^{\text {nd }}$ ICRC, id 654, Beijing, August, V. 11, 382-385, DOI: https://dx.doi.org/10.7529/ICRC2011/ V11/0654

Kobelev, P., Belov, A., Eroshenko, E., Yanke, V., 2013. Reception coefficients and energy characteristics of the ground level cosmic ray detectors. Proc 33rd ICRC, Rio de Janeiro, id 0878, http://spaceweather.izmiran.ru/descriptions/cc.pdf (last accessed April 8, 2021)

Korotkov, V., Berkova, M., Belov, A., Eroshenko, E., Kobelev, P., Yanke, V., 2011, "Effect of snow in cosmic ray variations and methods for taking it into consideration" //, Geomagnetism and Aeronomy, Volume 51, Issue 2, 247-253, DOI: https://dx.doi.org/10.1134/S0016793211020095

Korotkov, V., Berkova, M., Belov, A., Eroshenko, E., Yanke, V., Pyle, R., 2013, "Procedure to emend neutron monitor data that are affected by snow accumulations on and around the detector housing", JGR: Space Physics, Vol. 118, 6852-6857, DOI: https://dx.doi. org/10.1002/2013JA018647

Smith, S.W., 2002, Digital Signal Processing: A Practical Guide for Engineers and Scientists

Ventusky, Web-application for meteo data visualization, https://www.ventusky.com (last accessed November 23, 2020)

\section{Questions and answers}

Rolf Bütikofer: How do you determine the snow thickness and the density of the snow?

Answer: The method directly gives the thickness of the snow in terms of the amount of substance. We don't need snow density. Linear snow thickness is required to determine density.

Rolf Bütikofer: Yes, I know very well that it is difficult to determine the water equivalent of snow. But Rome NM is not ESOI NM.

Answer: The method directly gives the thickness of the snow in water equivalent. Yes, ESOI NM is not Rome NM or any other detector. The difference should be taken into account using the reception factors for the two detectors. But in order to minimize the error, it is desirable to use the closest detectors.

Christian T. Steigies: The variations could also come from wind, water vapour, or other things? Answer: Yes, wind accounting is especially important for high-latitude and mountain stations. 
Ludwig Klein: Do you have examples of wind corrections?

Answer: Yes, we dealt with the wind effect according to Mirny and Mawson data (using ground-based weather stations at the same points). However, the wind effect has not been reliably estimated: there is both a good correlation and its absence.

Kazuoki Munakata: I know only a correction using the free-air-pressure by nearby radiosonde measurements. Answer: Perhaps, but in this case, higher layers of the atmosphere create the wind effect. 